\title{
EIGENFUNCTIONS ARISING FROM A FIRST-ORDER FUNCTIONAL DIFFERENTIAL EQUATION IN A CELL GROWTH MODEL
}

\author{
BRUCE VAN BRUNT ${ }^{\bowtie 1}$ and M. VLIEG-HULSTMAN ${ }^{1}$
}

(Received 29 July, 2010; revised 21 December, 2010)

\begin{abstract}
A boundary-value problem for cell growth leads to an eigenvalue problem. In this paper some properties of the eigenfunctions are studied. The first eigenfunction is a probability density function and is of importance in the cell growth model. We sharpen an earlier uniqueness result and show that the distribution is unimodal. We then show that the higher eigenfunctions have nested zeros. We show that the eigenfunctions are not mutually orthogonal, but that there are certain orthogonality relations that effectively partition the set of eigenfunctions into two sets.
\end{abstract}

2000 Mathematics subject classification: primary 34K06; secondary 34K10.

Keywords and phrases: pantograph equation, cell growth model, nonlocal eigenvalue problem.

\section{Introduction}

The pantograph equation is

$$
y^{\prime}(x)+b y(x)=\lambda y(\alpha x),
$$

where $b, \lambda$ and $\alpha$ are constants. This equation arises in several applications such as light absorption in the Milky Way [2], a ruin problem [17], the collection of current from an electric train [15, 27] and cell growth [18, 20]. Versions of this equation for the retarded case $(|\alpha|<1)$ were studied earlier in the context of $q$-equations (see $[1,14])$. The analysis of solutions to the pantograph equation and the asymptotics of solutions as $x \rightarrow \infty$ is given in detail by Kato and McLeod [23] and also Iserles [22]. Complex versions of this equation were studied by Derfel and Iserles [13], among others, and using the work of Oberg [26] and Fredrickson [16], the behaviour of solutions in the complex plane was linked via complex dynamics to the existence of natural boundaries $[25,29,30]$.

\footnotetext{
${ }^{1}$ Institute of Fundamental Sciences, Mathematics, Massey University, Palmerston North, New Zealand; e-mail: b.vanbrunt@massey.ac.nz.

(C) Australian Mathematical Society 2011, Serial-fee code 1446-1811/2011 \$16.00
} 
The pantograph equation has been generalized in several ways. Heard [21] studied the equation with the functional argument $\alpha x$ replaced by the argument $x^{\alpha}$, and the complex version of this modified equation was studied in [28]. Second-order versions of the pantograph equation were studied in [24, 32, 33], and a matrix version was considered by Carr and Dyson [8]. More recently, attention has turned to pantograph equations with several functional arguments (see [7, 9]).

The pantograph equation is closely linked with problems in probability. Derfel [12] developed probability-based methods for studying and interpreting this equation. The applications to light absorption, the ruin problem and cell growth all require that the solution $y$ be a probability density function (pdf).

The original cell growth problem posed by Hall and Wake [18] is a boundary-value problem involving the pantograph equation

$$
y^{\prime}(x)+b y(x)=b \alpha y(\alpha x),
$$

where $b>0$ and $\alpha>1$. The solution is required to satisfy

$$
\lim _{x \rightarrow 0^{+}} y(x)=0, \quad \lim _{x \rightarrow \infty} y(x)=0,
$$

and since $y$ must also be a pdf, it is also required that $y(x) \geq 0$ for all $x \geq 0$, and that

$$
\int_{0}^{\infty} y(x) d x=1 .
$$

Hall and Wake [18] showed that this boundary-value problem is well posed: there exists a unique pdf solution. Da Costa et al. [11] showed that the solution must be unimodal. Properties of solutions have also been studied by Chang and Jau [10] and Begg [5]. The model has been applied to tumour growth [3] and the growth of plankton [4]. Recently, a multicompartment age-distribution model has been developed by Begg et al. [6].

The original cell growth model was extended by Hall and Wake [19] to include the case of exponential growth. In particular, the pantograph equation was generalized to the form

$$
y^{\prime}(x)+b x^{n} y(x)=b \alpha^{n+1} x^{n} y(\alpha x),
$$

where $n$ is a nonnegative integer, and it was shown that there is a unique pdf solution to the associated boundary-value problem. It is of interest to note that the condition $y \geq 0$ for $x \geq 0$ is nowhere imposed in the analysis of Hall and Wake for either boundaryvalue problem. Indeed, it was shown that the positivity of $y$ is a consequence.

The boundary-value problem can be recast as the eigenvalue problem

$$
y^{\prime}(x)+b x^{n} y(x)=\lambda \alpha^{n} x^{n} y(\alpha x),
$$

where $b>0, \alpha>1$ are constants, $n$ is a nonnegative integer, and $\lambda$ is a nonzero eigenvalue parameter. The normalizing condition (1.2) is still imposed, but the requirement of positivity for solutions is dropped. This problem was studied in [31], where it was shown that there exists an infinite set of eigenvalues, $\left\{\lambda_{m}\right\}$, and a 
corresponding set of eigenfunctions, $\left\{y_{m}\right\}$. The first eigenfunction in this analysis corresponds to the pdf solutions obtained by Hall and Wake $[18,19]$. The eigenvalues are given by

$$
\lambda_{m}=b \alpha^{m(n+1)+1},
$$

for $m=0,1,2, \ldots$, and the eigenfunctions are given by

$$
y_{m}(x)=K_{m}\left(e^{-b x^{n+1} /(n+1)}+\sum_{k=1}^{\infty} p_{k}\left(\lambda_{m}\right) e^{-b \alpha^{k(n+1)} x^{n+1} /(n+1)}\right),
$$

where

$$
K_{m}=(n+1)\left(\frac{b}{n+1}\right)^{1 /(n+1)}\left(\Gamma\left(\frac{1}{n+1}\right)\right)^{-1} \prod_{k=0}^{\infty}\left(1-\frac{1}{b \alpha^{(k-m)(n+1)+1}}\right)^{-1}
$$

and

$$
p_{k}\left(\lambda_{m}\right)=\frac{(-1)^{k} \alpha^{k m(n+1)}}{\alpha^{(n+1) k(k-1) / 2} \prod_{j=1}^{k}\left(1-\alpha^{-(n+1) j)}\right.} .
$$

In this paper we study some of the properties of these eigenfunctions, particularly the zeros and the location of relative extrema. We show that in some respects, such as the distribution of zeros for eigenfunctions, the problem behaves like the classical singular Sturm-Liouville problem. In other respects, however, the problem differs. The eigenfunctions, for instance, do not form a mutually orthogonal set under the weight function analogous to that for the Sturm-Liouville problem. Intriguingly, the eigenfunctions do satisfy certain orthogonality relations that partition them into two classes.

The first eigenfunction $y_{0}$ is of particular interest because it corresponds to a pdf in the cell growth model [19]. In the next section, we sharpen the uniqueness result given in $[19,31]$ for this case. We then study analytically the shape of the solution and show that the distribution must be unimodal. Some of the results of Section 2 can be adapted to prove similar results for the higher eigenfunctions. In Section 3 we study properties of the higher eigenfunctions, including the zeros and orthogonality relations.

\section{The first eigenfunction}

Let $\lambda=\lambda_{0}=b \alpha$. It was shown in [31] (and in [19]) that the series (1.5) defining the first eigenfunction $y_{0}$ is a pdf that solves the boundary-value problem. In fact, it was shown that $y_{0}(x)>0$ for all $x>0$. It was also shown that if the class of solutions is limited to functions $y$ such that

$$
\int_{0}^{\infty} x^{n} y(x) d x<\infty
$$

then the solution $y_{0}$ is unique. The cell growth problem, however, makes sense without condition (2.1). Indeed, condition (1.2) is the only integrability condition that can 
be imposed naturally from this model, and this raises the question of whether there are pdf solutions to the boundary-value problem that do not satisfy (2.1). Here, we are concerned with classical solutions to the problem that are at least continuously differentiable on $(0, \infty)$ and continuous on $[0, \infty)$. The next result sharpens the earlier uniqueness results and shows that any nonnegative solution to the boundaryvalue problem must satisfy condition (2.1), and that the Dirichlet series solution (1.5) is therefore unique.

THEOREM 2.1. Let $\lambda=b \alpha$. Then there exists a unique solution to the boundary-value problem (1.3) subject to (1.2) that is nonnegative on $[0, \infty)$.

PROOF. Equation (1.3) with $\lambda=b \alpha$ can be integrated to get

$$
y(x)=b \int_{x}^{\alpha x} \xi^{n} y(\xi) d \xi .
$$

Note that the condition $y(x) \rightarrow 0$ as $x \rightarrow \infty$ implies that

$$
\lim _{x \rightarrow \infty} \int_{x}^{\alpha x} \xi^{n} y(\xi) d \xi=0
$$

but this relation does not yield the Cauchy criterion.

Suppose that $y$ is a nonnegative solution to the boundary-value problem. Condition (1.2) implies that $y \in L^{1}[0, \infty)$, and integrating (2.2) from 0 to $\infty$ gives

$$
\int_{0}^{\infty} y(\xi) d \xi=b \int_{0}^{\infty} \int_{\xi}^{\alpha \xi} \tau^{n} y(\tau) d \tau d \xi
$$

Since $y$ is nonnegative, the double integral must be absolutely convergent. Fubini's theorem shows that the order of integration can be changed; hence,

$$
\int_{0}^{\infty} y(\xi) d \xi=b \int_{0}^{\infty} \int_{\tau / \alpha}^{\tau} \tau^{n} y(\tau) d \xi d \tau=b \int_{0}^{\infty}\left(1-\frac{1}{\alpha}\right) \tau^{n+1} y(\tau) d \tau .
$$

Condition (1.2) therefore shows that

$$
\int_{0}^{\infty} x^{n+1} y(x) d x=\frac{\alpha}{b(\alpha-1)},
$$

and the comparison test thus shows that condition (2.1) must be satisfied. The uniqueness of the solution thus follows by [31, Theorem 3.2].

The remainder of this section is devoted to showing analytically that the probability distribution $y_{0}$ is unimodal. This result is also useful for studying the zeros of higher eigenfunctions. We note that even for the simplest case $(n=0)$, the unimodal character of the distribution has yet to be established, although the graphs of solutions strongly suggest the result (see [18, 19]). The proof is somewhat lengthy and we break it down into smaller, more digestible lemmas. We show a stronger result, namely that $y^{\prime}(x)=0$ has a unique positive solution. 
LEMMA 2.2. The equation

$$
y_{0}^{\prime}(x)=0
$$

has a finite number of positive solutions.

ProOF. We show first that the zeros of $y_{0}$ on $[0, \infty)$ must be isolated. The function $y_{0}$ can be regarded as a complex-valued function of a complex variable $z$. The Dirichlet series that defines $y_{0}(z)$ is uniformly convergent in any compact subset of the half plane $\Pi_{0}=\{z: \operatorname{Re}(z)>0\}$. Weierstrass' theorem shows that $y_{0}$ is holomorphic in $\Pi_{0}$; consequently, $y_{0}^{\prime}$ is also holomorphic in $\Pi_{0}$. Conditions (1.1) and (1.2) preclude $y_{0}$ from being a constant function, and therefore $y_{0}^{\prime}$ is not identically zero in $\Pi_{0}$. The identity theorem implies that the zeros of $y_{0}^{\prime}$ must be isolated, so that in particular the zeros of $y_{0}^{\prime}$ that occur on the positive real axis must be isolated.

The above argument does not include the origin since $y_{0}^{\prime}$ need not be holomorphic there. It may be that the origin is a limit point for zeros of $y_{0}^{\prime}$. To eliminate this possibility, we note that $y_{0}(x)>0$ for all $x>0$, and $y(0)=0$. The function $y_{0}^{\prime}$ is continuous on $[0, \infty)$, and hence there must be a number $x_{1}>0$ such that $y_{0}^{\prime}(x)>0$ for all $x \in\left(0, x_{1}\right)$. All the zeros of $y_{0}^{\prime}$ on $[0, \infty)$ must therefore be isolated.

Equation (1.5) shows that the derivative of the solution $y_{0}$ is

$$
y_{0}^{\prime}(x)=-b K_{0} x^{n}\left(e^{-b x^{n+1} /(n+1)}+\sum_{k=1}^{\infty} g_{k}(x)\right),
$$

where $b>0, K_{0}>0$ and

$$
g_{k}(x)=O\left(e^{-b \alpha^{k(n+1)} x^{n+1} /(n+1)}\right)
$$

as $x \rightarrow \infty$. We thus have

$$
y_{0}^{\prime}(x) \sim-b K_{0} x^{n} e^{-b x^{n+1} /(n+1)}
$$

as $x \rightarrow \infty$, and consequently there is an $\hat{x}>0$ such that $y_{0}^{\prime}(x)<0$ for all $x>\hat{x}$. The positive zeros of $y_{0}^{\prime}$ must therefore lie in the interval $[0, \hat{x}]$. Since there are no limit points for zeros in this interval, the Bolzano-Weierstrass theorem implies that the number of zeros must be finite. The function $y_{0}$ is smooth and positive on $(0, \infty)$, and condition (1.1) implies that $y_{0}$ must have at least one local maximum in this interval. Therefore, there is at least one positive solution to equation (2.3).

Lemma 2.3. Suppose that equation (2.3) has at least two positive solutions. Then there is a solution $\xi$ to equation (2.3) such that $\xi>M_{1}$, where $M_{1}$ denotes the smallest positive value at which $y_{0}$ has a local maximum.

PROOF. Lemma 2.2 shows that any positive solutions to equation (2.3) must be isolated and that $y_{0}$ must have at least one local maximum; consequently, there is a smallest positive value $M_{1}$ at which $y_{0}$ has a local maximum. If $x>0$ and $y_{0}^{\prime}(x)=0$, then equation (1.3) implies that

$$
y_{0}(x)=\alpha^{n+1} y_{0}(\alpha x) .
$$


Differentiating equation (1.3) gives

$$
y_{0}^{\prime \prime}(x)+b(n-1) x^{n-1}\left(y_{0}(x)-\alpha^{n+1} y_{0}(\alpha x)\right)=b x^{n}\left(\alpha^{n+2} y_{0}^{\prime}(\alpha x)-y_{0}^{\prime}(x)\right),
$$

and equation (2.4) implies that

$$
y_{0}^{\prime \prime}(x)=b \alpha^{n+2} x^{n} y_{0}^{\prime}(\alpha x) .
$$

Suppose that there are two positive solutions to equation (2.3). Then there is an $\eta \neq M_{1}$ such that $y_{0}^{\prime}(\eta)=0$. If $\eta>M_{1}$, then let $\xi=\eta$. Suppose that $0<\eta<M_{1}$. The definition of $M_{1}$ precludes any local extrema in $\left(0, M_{1}\right)$; hence, $y_{0}^{\prime \prime}(\eta)=0$. Equation (2.5) shows that $y_{0}^{\prime}(\alpha \eta)=0$. Since $y_{0}$ is positive for all $x>0$ and $\alpha>1$, equation (2.4) implies $y_{0}(\eta)>y_{0}(\alpha \eta)$; consequently, there must be a local maximum between $\eta$ and $\alpha \eta$. Given that $\eta<M_{1}$, we have $\alpha \eta>M_{1}$, so that we can choose $\xi=\alpha \eta$.

LEMma 2.4. Suppose that $y_{0}$ has a local maximum at $M$ and that there is a solution $\xi$ to equation (2.3) such that $\xi>M$. Then there is an $m>M$ at which $y_{0}$ has a local minimum.

PROOF. We first show that there is a $\tau>M$ such that $y_{0}^{\prime}(\tau)=0$ and $y_{0}^{\prime \prime}(\tau) \neq 0$. Suppose that there is no such point. Then $y_{0}^{\prime}(\xi)=y_{0}^{\prime \prime}(\xi)=0$, and equation (2.5) implies that $y_{0}^{\prime}(\alpha \xi)=0$. Therefore $y_{0}^{\prime}(\alpha \xi)=y_{0}^{\prime \prime}(\alpha \xi)=0$, so that $y_{0}^{\prime}\left(\alpha^{2} \xi\right)=0$. It is clear that this argument can be repeated to establish an infinite sequence $\left\{\alpha^{k} \xi\right\}$ of points that are solutions to equation (2.3). This, however, contradicts Lemma 2.2. So there must be a $\tau>M$ such that $y_{0}^{\prime}(\tau)=0$ and $y_{0}^{\prime \prime}(\tau) \neq 0$.

If $y_{0}^{\prime \prime}(\tau)>0$, then we can take $m=\tau$. If $y_{0}^{\prime \prime}(\tau)<0$, then $\tau$ corresponds to a local maximum and hence there must be a local minimum at some point $m$ between $M$ and $\tau$.

THEOREM 2.5. There exists a unique positive solution to equation (2.3). In particular, the probability distribution function $y_{0}$ is unimodal.

PROOF. Suppose that equation (2.3) has at least two positive solutions. Lemma 2.3 implies that there is a $\xi_{1}>M_{1}$ that solves equation (2.3). Lemma 2.4 thus implies that $y_{0}$ has a local minimum at some point $m_{1}>M_{1}$. Since $y_{0}$ is positive and goes to zero as $x \rightarrow \infty$, there must be another local maximum beyond $m_{1}$. Let $M_{2}$ denote the closest point beyond $m_{1}$ at which $y_{0}$ has a local maximum. Then $y_{0}^{\prime \prime}\left(m_{1}\right) \geq 0$ and $y_{0}^{\prime \prime}\left(M_{2}\right) \leq 0$. Equation (2.5) implies that $y_{0}^{\prime}\left(\alpha m_{1}\right) \geq 0$ and $y_{0}^{\prime}\left(\alpha M_{2}\right) \leq 0$. The continuity of $y_{0}^{\prime}$ thus indicates that there is a solution $\xi_{2}$ to equation (2.3) in the interval $\left[\alpha m_{1}, \alpha M_{2}\right]$. Equation (2.4), however, gives $y_{0}\left(m_{1}\right)>y_{0}\left(\alpha m_{1}\right)$, and therefore $y_{0}$ must have a local maximum between $m_{1}$ and $\alpha m_{1}$. The definition of $M_{2}$ implies that $M_{2}<\alpha m_{1}$; consequently, $\xi_{2}>M_{2}$.

Lemma 2.4 can now be applied to $M_{2}$ and $\xi_{2}$ to establish the existence of another local minimum at some point $m_{2}>M_{2}$, and the argument used above can be applied to show that there is another local maximum at some point $M_{3}>m_{2}$ and a point $\xi_{3}>M_{3}$ 
that solves equation (2.3). It is clear that we can repeat this argument to establish the existence of an infinite sequence $\left\{\xi_{k}\right\}$ of points that are solutions to equation (2.3) such that $\xi_{k} \rightarrow \infty$ as $k \rightarrow \infty$. The existence of such a sequence, however, contradicts Lemma 2.2. We thus conclude that equation (2.3) has a unique positive solution.

The above result shows that $y_{0}$ has a unique local maximum in $(0, \infty)$. The next result gives bounds for the location of this maximum.

THEOREM 2.6. Let $M$ denote the position of the maximum for $y_{0}$ in $(0, \infty)$. Then

$$
v \leq M \leq \alpha v,
$$

where

$$
v=\left(\frac{n+1}{b\left(\alpha^{n+1}-1\right)}\right)^{1 /(n+1)} .
$$

PROOF. Equation (2.2) gives

$$
y_{0}(M)=b \int_{M}^{\alpha M} \xi^{n} y_{0}(\xi) d \xi
$$

consequently,

$$
y_{0}(M) \leq b y_{0}(M) \int_{M}^{\alpha M} \xi^{n} d \xi=b y_{0}(M)\left(\alpha^{n+1}-1\right) \frac{M^{n+1}}{n+1} .
$$

We thus get $v \leq M$. Since $y_{0}$ is unimodal, $y_{0}(M / \alpha) \leq y_{0}(x)$ for all $x \in[M / \alpha, M]$. Equation (2.2) therefore yields

$$
y_{0}\left(\frac{M}{\alpha}\right)=b \int_{M}^{\alpha M} \xi^{n} y_{0}(\xi) d \xi \geq b y_{0}\left(\frac{M}{\alpha}\right)\left(1-\frac{1}{\alpha^{n+1}}\right) \frac{M^{n+1}}{n+1},
$$

which gives $M \leq \alpha \nu$.

\section{Higher eigenfunctions}

In this section we look at some elementary properties of the eigenfunctions $y_{m}$ for $m \geq 1$. We first show that the set of eigenfunctions is linearly independent, and then we study the distribution of zeros and orthogonality properties.

THEOREM 3.1. The set $\left\{y_{m}\right\}$ is linearly independent.

Proof. Suppose that the set is linearly dependent. Then there exist $m \in \mathbb{N} \cup\{0\}$, $N \in \mathbb{N}, m_{k} \in \mathbb{N} \cup\{0\}$ and $\beta_{k} \in \mathbb{R}, k=1,2, \ldots, N$, such that $m \neq m_{k}$ for all $k$, at least one of the $\beta_{k}$ is nonzero, and

$$
y_{m}(x)=\sum_{k=1}^{N} \beta_{k} y_{m_{k}}(x) .
$$


Substituting the above expression into equation (1.3) with $\lambda=\lambda_{m}$ gives

$$
y_{m}^{\prime}(x)+b x^{n} y_{m}(x)=\lambda_{m} \alpha^{n} x^{n} y_{m}(\alpha x)=\lambda_{m} \alpha^{n} x^{n} \sum_{k=1}^{N} \beta_{k} y_{m_{k}}(\alpha x),
$$

and the definition of $y_{m_{k}}$ gives

$$
y_{m}^{\prime}(x)+b x^{n} y_{m}(x)=\sum_{k=1}^{N} \beta_{k}\left(y_{m_{k}}^{\prime}(x)+b x^{n} y_{m_{k}}(x)\right)=\sum_{k=1}^{N} \beta_{k} \lambda_{m_{k}} \alpha^{n} x^{n} y_{m_{k}}(\alpha x) ;
$$

consequently,

$$
\sum_{k=1}^{N} \beta_{k}\left(\lambda_{m}-\lambda_{m_{k}}\right) y_{m_{k}}(x)=0
$$

Now, $\lambda_{m}-\lambda_{m_{k}} \neq 0$ for all $k$, and at least one of the $\beta_{k}$ is nonzero. We thus conclude that the set $\left\{y_{m_{k}}\right\}$ is linearly dependent. The above argument can be repeated $N-1$ times to show that there is an $m_{j}, 1 \leq j \leq N$, such that $y_{m}(x)=B y_{m_{j}}(x)$, where $B \neq 0$. This now yields

$$
B\left(\lambda_{m}-\lambda_{m_{j}}\right) y_{m_{j}}(x)=0 .
$$

Since $y_{m_{j}}$ is not identically zero, we conclude that $y_{m}$ cannot be represented as a finite linear combination of other eigenfunctions.

We now turn to the zeros of the eigenfunctions. Let $\delta_{1}$ be the operator defined by

$$
\delta_{1} y(x)=\int_{x}^{\infty} \xi^{n} y(\xi) d \xi .
$$

The operator $\delta_{1}$ was introduced in [31] as a tool to prove uniqueness of the eigenfunctions among a class of rapidly decaying functions. The eigenfunctions are closely related through this operator.

THEOREM 3.2. For all $m \geq 1$ and $x \geq 0$,

$$
-x^{n} y_{m}(x)=\frac{K_{m}}{b K_{m-1}} y_{m-1}^{\prime}(x) \text {. }
$$

PROOF. The eigenfunctions are defined by the Dirichlet series in equation (1.5). The uniform convergence of this series indicates that it can be integrated term by term. We thus get

$$
\delta_{1} y_{m}(x)=\frac{K_{m}}{b}\left(e^{-b x^{n+1} /(n+1)}+\sum_{k=1}^{\infty} \frac{p_{k}\left(\lambda_{m}\right)}{\alpha^{k(n+1)}} e^{-b \alpha^{k(n+1)} x^{n+1} /(n+1)}\right) .
$$

Equations (1.4) and (1.7) yield

$$
\frac{p_{k}\left(\lambda_{m}\right)}{\alpha^{k(n+1)}}=p_{k}\left(\lambda_{m-1}\right)
$$


and therefore

$$
\delta_{1} y_{m}(x)=\frac{K_{m}}{b K_{m-1}} y_{m-1}(x) .
$$

Differentiating both sides of equation (3.2) gives equation (3.1).

Equation (3.1) and Theorem 2.5 imply that $y_{1}$ has only one positive zero. Let $z_{1,1}$ denote this zero. Then $y_{1}(x)>0$ for $x \in\left(0, z_{1,1}\right)$, and $y_{1}(x)<0$ for $x \in\left(z_{1,1}, \infty\right)$; hence, $y_{1}$ must have a local maximum in $\left(0, z_{1,1}\right)$ and a local minimum in $\left(z_{1,1}, \infty\right)$. This means that $y_{2}$ must have a zero in each of these intervals. It may be, however, that $y_{1}$ has other local extrema, each of which would correspond to a zero for $y_{2}$. The next result precludes this possibility.

THEOREM 3.3. There exist precisely two positive solutions to $y_{2}(x)=0$. Let $z_{2,1}$ and $z_{2,2}$ denote these solutions, with $z_{2,1}<z_{2,2}$. Then

$$
0<z_{2,1}<\frac{z_{1,1}}{\alpha}<z_{1,1}<z_{2,2}
$$

PROOF. The proof is along very similar lines to that of Theorem 2.5. In fact, the arguments leading up to Theorem 2.5 can be applied to $y_{1}$ to show that $y_{1}^{\prime}(x)=0$ has only one solution in $\left(z_{1,1}, \infty\right)$. We thus focus on the zeros of $y_{1}^{\prime}$ in $\left(0, z_{1,1}\right)$.

The function $y_{1}$ must have at least one local maximum in $\left(0, z_{1,1}\right)$. Let $M_{1,1}$ be the smallest value in this interval at which $y_{1}$ has a local maximum. Suppose that there is a point $w \neq M_{1,1}$ in this interval such that $y^{\prime}(w)=0$. For any $x>0$ such that $y^{\prime}(x)=0$, the analogues of equations (2.4) and (2.5) are

$$
\begin{aligned}
& y_{1}(x)=\alpha^{2(n+1)} y_{1}(\alpha x), \\
& y_{1}^{\prime \prime}(x)=b \alpha^{2 n+3} y_{1}^{\prime}(\alpha x) .
\end{aligned}
$$

In particular, $y_{1}(w)>0$ and therefore, by equation (3.4), $y_{1}(\alpha w)>0$. We thus have that $\alpha w<z_{1,1}$. The arguments used to establish Lemma 2.3 can be used to show that there is a $\xi>M_{1,1}$ such that $y^{\prime}(\xi)=0$, and the construction in the proof of Lemma 2.4 can then be used to establish the existence of a local minimum between $M_{1,1}$ and $z_{1,1}$.

Without loss of generality, we can assume that $w$ corresponds to the largest value in $\left(0, z_{1,1}\right)$ such that $y_{1}$ has a local minimum. Equation (3.4) implies that $y_{1}(w)>y(\alpha w)>0$; hence, there is a local maximum at some point $\sigma \in(w, \alpha w)$. The definition of $w$ implies that $\sigma$ must correspond to the largest value in $\left(0, z_{1,1}\right)$ at which $y_{1}$ has a local maximum. Since $w$ corresponds to a local minimum, equation (3.5) implies that $y_{1}^{\prime}(\alpha w) \geq 0$. Similarly, we have $y_{1}^{\prime}(\alpha \sigma) \geq 0$, so that $y_{1}^{\prime}$ must have a zero at some point $\tau \in(\alpha w, \alpha \sigma)$. There are no local extrema in this interval and therefore $y_{1}^{\prime \prime}(\tau)=0$ so that, by equation (3.5), $y_{1}^{\prime}(\tau)=0$. We can repeat this argument any number of times to get a sequence $\left\{\alpha^{k} \tau\right\}$ such that $y_{1}^{\prime}\left(\alpha^{k} \tau\right)=0$ and $\alpha^{k} \tau<z_{1,1}$. For $k$ sufficiently large, however, $\alpha^{k} \tau>z_{1,1}$. This contradiction shows that $y^{\prime}(x)$ has only one zero $z_{2,1}=M_{1,1}$ in $\left(0, z_{1,1}\right)$. Since $\alpha z_{2,1}<z_{1,1}$ we have $z_{2,1}<z_{1,1} / \alpha$. Finally, note that if $y_{1}^{\prime}\left(z_{1,1}\right)=0$, then equation (1.3) implies that $y_{1}\left(\alpha z_{1,1}\right)=0$ and therefore, by Theorem 3.2, $y_{0}^{\prime}$ has at least two zeros, which contradicts Theorem 2.5. 
It is clear that the above arguments can be modified to show, for example, that $y_{3}$ has precisely three positive zeros $z_{3,1}, z_{3,2}, z_{3,3}$, and if $z_{3,1}<z_{3,2}<z_{3,3}$, then

$$
z_{3,1}<\frac{z_{2,1}}{\alpha}<z_{2,1}<z_{3,2}<\frac{z_{2,2}}{\alpha}<z_{2,2}<z_{3,3}
$$

and that the pattern continues throughout the higher eigenfunctions. In summary, the following result can be established.

THEOREM 3.4. The function $y_{m}$, where $m \geq 1$, has precisely $m$ positive zeros. These zeros correspond to local extrema for $y_{m-1}$. The zeros of two consecutive eigenfunctions are nested: if $z_{m-1,1}, z_{m-1,2}, \ldots, z_{m-1, m-1}$ and $z_{m, 1}, z_{m, 2}, \ldots, z_{m, m}$ denote the zeros of $y_{m-1}$ and $y_{m}$ respectively, each arranged in ascending magnitude, then

$$
z_{m, 1}<z_{m-1,1}<z_{m, 2}<z_{m-1,2}<\cdots<z_{m, m-1}<z_{m-1, m-1}<z_{m, m} .
$$

Moreover, for $j=1,2, \ldots, m-1$,

$$
z_{m, j}<\frac{z_{m-1, j}}{\alpha}
$$

The above theorem shows that the position of the $j$ th zero decreases rapidly as $m$ increases. In particular, for $m>1$, the position of the first zero of $y_{m}$ satisfies

$$
z_{m, 1}<\frac{z_{1,1}}{\alpha^{m-1}}
$$

Since $z_{1,1}=M$ in Theorem 2.6,

$$
z_{m, 1}<\frac{v}{\alpha^{m-2}} .
$$

Sturm-Liouville problems in Hilbert spaces produce orthogonal families of eigenfunctions, and it is natural to enquire whether the eigenfunctions defined by equation (1.5) are orthogonal. For the boundary-value problem (1.3) subject to (1.2), the weight function for the inner product is $x^{n}$. Define the inner product by

$$
\langle f, g\rangle=\int_{0}^{\infty} x^{n} f(x) g(x) d x .
$$

Note that, for all $n, m \geq 0, y_{m}$ is a smooth function for $x \geq 0$. Evidently,

$$
y_{m}(x) \sim K_{m} e^{-b x^{n+1} /(n+1)}
$$

as $x \rightarrow \infty$; hence,

$$
y_{m}^{2}(x) \sim K_{m}^{2} e^{-2 b x^{n+1} /(n+1)}
$$

as $x \rightarrow \infty$. We thus see that

$$
\left\langle y_{m}, y_{m}\right\rangle<\infty
$$


so that the eigenfunctions can be regarded as elements of the real (weighted) Hilbert space $L^{2}\left([0, \infty), x^{n} d x\right)$. Similar arguments show that

$$
\left\langle y_{m}, y_{j}\right\rangle<\infty
$$

for all $m, j \geq 0$.

It turns out that the eigenfunctions do not form a mutually orthogonal family under the above inner product; nonetheless, there are certain orthogonality relations that partition the eigenfunctions into two sets.

Theorem 3.5. For all $m \geq 0$,

$$
\begin{gathered}
\left\langle y_{m}, y_{m+1}\right\rangle=0, \\
\left\langle y_{m}, y_{m+2}\right\rangle \neq 0 .
\end{gathered}
$$

Proof. We first establish relation (3.6). Let $u=y_{m}$ and $d v=x^{n} y_{m+1} d x$. Then $d u=y_{m}^{\prime}$ and $v=-\delta_{1} y_{m+1}$; hence integration by parts yields

$$
\left\langle y_{m}, y_{m+1}\right\rangle=\int_{0}^{\infty} y_{m}^{\prime}(x) \delta_{1} y_{m+1}(x) d x
$$

where the boundary terms vanish by condition (1.1). Equation (3.2) gives

$$
\left\langle y_{m}, y_{m+1}\right\rangle=\frac{K_{m+1}}{b K_{m}} \int_{0}^{\infty} y_{m}^{\prime}(x) y_{m}(x) d x=\left.\frac{K_{m+1}}{2 b K_{m}} y_{m}^{2}(x)\right|_{0} ^{\infty}=0 .
$$

Relation (3.7) follows similarly. Let $u=y_{m}$ and $d v=x^{n} y_{m+2} d x$. Then

$$
d u=y_{m}^{\prime} d x=-\frac{b K_{m}}{K_{m+1}} x^{n} y_{m+1} d x
$$

and

$$
v=-\delta_{1} y_{m+2}=-\frac{K_{m+1}}{b K_{m}} y_{m+1}
$$

hence

$$
\begin{aligned}
\left\langle y_{m}, y_{m+2}\right\rangle & =-\left.\frac{K_{m+1}}{b K_{m}} y_{m}(x) y_{m+1}(x)\right|_{0} ^{\infty}-\int_{0}^{\infty} x^{n} y_{m+1}^{2}(x) d x \\
& =-\int_{0}^{\infty} x^{n} y_{m+1}^{2}(x) d x \neq 0 .
\end{aligned}
$$

Corollary 3.6. For all $m \geq 0$, if $j \in \mathbb{N}$ is odd, then

$$
\left\langle y_{m}, y_{m+j}\right\rangle=0
$$

if $j \in \mathbb{N}$ is even, then

$$
\left\langle y_{m}, y_{m+j}\right\rangle \neq 0
$$


PROOF. Integration by parts gives

$$
\left\langle y_{m}, y_{m+j}\right\rangle=-\frac{K_{m} K_{m+j}}{K_{m+1} K_{m+j-1}}\left\langle y_{m}, y_{m+j-1}\right\rangle .
$$

If $j=2 k+1$, then the above relation can be applied $k$ times to get

$$
\left\langle y_{m}, y_{m+j}\right\rangle=\Lambda_{m, j}\left\langle y_{m+k}, y_{m+j-k}\right\rangle=\Lambda_{m, j}\left\langle y_{m+k}, y_{m+k+1}\right\rangle=0,
$$

where $\Lambda_{m, j}$ is a nonzero constant formed by the constants $K_{i}$. If $j=2 k$, then relation (3.8) can be applied $k$ times to get

$$
\left\langle y_{m}, y_{m+j}\right\rangle=\hat{\Lambda}_{m, j}\left\langle y_{m+k}, y_{m+k}\right\rangle,
$$

where $\hat{\Lambda}_{m, j}$ is also a nonzero constant formed by the constants $K_{i}$. The above relation shows that $\left\langle y_{m}, y_{m+j}\right\rangle \neq 0$.

\section{References}

[1] C. R. Adams, "Linear q-difference equations", Bull. Amer. Math. Soc. 37 (1931) 361-400.

[2] V. A. Ambartsumian, "On the fluctuation of brightness of the Milky Way", Dokl. Akad. Nauk USSR 44 (1944) 223-226.

[3] B. Basse, B. Baguley, E. Marshall, W. Joseph, B. van Brunt, G. C. Wake and D. J. N. Wall, "Modelling cell death in human tumour cell lines exposed to the anticancer drug Paclitaxel", J. Math. Biol. 49 (2004) 329-357.

[4] B. Basse, G. C. Wake, D. J. N. Wall and B. van Brunt, "On a cell-growth model for plankton", IMA J. Math. Med. Biol. 21 (2004) 49-61.

[5] R. Begg, "Existence theorems for a class of nonlocal differential equations", J. Math. Anal. Appl. 322 (2006) 1168-1187.

[6] R. Begg, D. J. N. Wall and G. C. Wake, "On a multicompartment age-distribution model of cell growth", IMA J. Appl. Math. 75 (2010) 905-931.

[7] L. Bogachev, G. Derfel, S. Molchanov and J. R. Ockendon, "On bounded solutions of the balanced generalized pantograph equation", in: Topics in stochastic analysis and nonparametric estimation, Volume 145 of IMA Volumes in Mathematics and its Applications (eds P. Chow, B. Mordukhovich and G. Yin), (Springer, New York, 2008) 29-49.

[8] J. Carr and J. Dyson, "The matrix functional differential equation $y^{\prime}(x)=A y(\lambda x)+B y(x)$ ", Proc. Roy. Soc. Edinburgh A 75 (1975) 5-22.

[9] J. Čermák, "On matrix differential equations with several unbounded delays", European J. Appl. Math. 17 (2006) 417-433.

[10] Y. H. Chang and G. C. Jau, "The behaviour of the solution for a mathematical model for analysis of the cell cycle", Commun. Pure Appl. Anal. 5 (2006) 779-792.

[11] F. P. da Costa, M. Grinfeld and J. B. McLeod, "Unimodality of steady size distributions of growing cell populations", J. Evol. Equ. 1 (2001) 405-409.

[12] G. Derfel, "Probabilistic method for a class of functional differential equations", Ukraïn. Mat. Zh. 41 (1989) 1322-1327; English translation: Ukrainian Math. J. 41 (1990) 1137-1141.

[13] G. Derfel and A. Iserles, "The pantograph equation in the complex plane", J. Math. Anal. Appl. 213 (1997) 117-132.

[14] P. Flamant, "Sur une équation différentielle fonctionnelle linéaire", Palermo Rend. 48 (1924) 135-208.

[15] L. Fox, D. F. Mayers, J. R. Ockendon and A. B. Tayler, "On a functional differential equation", J. Inst. Math. Appl. 8 (1971) 271-307. 
[16] P. O. Fredrickson, "Dirichlet series solution for certain functional differential equations", in: Japan-United States seminar on ordinary differential and functional equations, Volume 243 of Springer Lecture Notes in Mathematics (ed. M. Uradbe), (Springer, New York, 1971) 247-254.

[17] D. P. Gaver, "An absorption probablity problem”, J. Math. Anal. Appl. 9 (1964) 384-393.

[18] A. J. Hall and G. C. Wake, "A functional differential equation arising in the modelling of cellgrowth”, J. Aust. Math. Soc. Ser. B 30 (1989) 424-435.

[19] A. J. Hall and G. C. Wake, "Functional differential equations determining steady size distributions for populations of cells growing exponentially", J. Aust. Math. Soc. Ser. B 31 (1990) 434-453.

[20] A. J. Hall, G. C. Wake and P. W. Gandar, "Steady size distributions for cells in one dimensional plant tissues", J. Math. Biol. 30 (1991) 101-123.

[21] M. Heard, "Asymptotic behaviour of solutions of the functional differential equation $x^{\prime}(t)=$ $a x(t)+b x\left(t^{\alpha}\right), \alpha>1$ ", J. Math. Anal. Appl. 44 (1973) 745-757.

[22] A. Iserles, "On the generalized pantograph functional-differential equation", European J. Appl. Math. 4 (1993) 1-38.

[23] T. Kato and J. B. McLeod, "The functional differential equation $y^{\prime}(x)=a y(\lambda x)+b y(x)$ ", Bull. Amer. Math. Soc. 77 (1971) 891-937.

[24] H. K. Kim, "Advanced second order functional differential equations", Ph. D. Thesis, Massey University, 1998.

[25] J. C. Marshall, B. van Brunt and G. C. Wake, "Natural boundaries for solutions to a certain class of functional differential equations", J. Math. Anal. Appl. 268 (2002) 157-170.

[26] R. J. Oberg, "Local theory of complex functional differential equations", Trans. Amer. Math. Soc. 161 (1971) 302-327.

[27] J. R. Ockendon and A. B. Tayler, "The dynamics of a current collection system for an electric locomotive", Proc. R. Soc. Lond. A 322 (1971) 447-468.

[28] B. van Brunt, H. O. Kim and G. Derfel, "Holomorphic solutions to functional differential equations", J. Math. Anal. Appl. 368 (2010) 350-357.

[29] B. van Brunt, J. C. Marshall and G. C. Wake, "An eigenvalue problem for holomorphic solutions to a certain class of functional differential equations", European J. Appl. Math. 14 (2003) 571-585.

[30] B. van Brunt, J. C. Marshall and G. C. Wake, "Holomorphic solutions to pantograph type equations with neutral fixed points", J. Math. Anal. Appl. 295 (2004) 557-569.

[31] B. van Brunt and M. Vlieg-Hulstman, "An eigenvalue problem involving a functional differential equation arising in a cell growth model", ANZIAM J. 51 (2010) 383-393.

[32] B. van Brunt, G. C. Wake and H. K. Kim, "On a singular Sturm-Liouville problem involving an advanced functional differential equation", European J. Appl. Math. 12 (2001) 625-644.

[33] G. C. Wake, S. Cooper, H. K. Kim and B. van Brunt, "Functional differential equations for cellgrowth models with dispersion", Commun. Appl. Anal. 4 (2000) 561-573. 